Research Article

\title{
A Strong Law of Large Numbers for Random Sets in Fuzzy Banach Space
}

\author{
R. Ghasemi, A. Nezakati, and M. R. Rabiei $i$ \\ Faculty of Mathematical Sciences, Shahrood University of Technology, Shahrood, Iran \\ Correspondence should be addressed to M. R. Rabiei; rabie1354@yahoo.com
}

Received 14 December 2019; Revised 24 February 2020; Accepted 25 May 2020; Published 10 August 2020

Academic Editor: Katsuhiro Honda

Copyright (c) 2020 R. Ghasemi et al. This is an open access article distributed under the Creative Commons Attribution License, which permits unrestricted use, distribution, and reproduction in any medium, provided the original work is properly cited.

\begin{abstract}
The main purpose of this paper is to consider the strong law of large numbers for random sets in fuzzy metric space. Since many years ago, limited theorems have been expressed and proved for fuzzy random variables, but despite the uncertainty in fuzzy discussions, the nonfuzzy metric space has been used. Given that the fuzzy random variable is defined on the basis of random sets, in this paper, we generalize the strong law of large numbers for random sets in the fuzzy metric space. The embedded theorem for compact convex sets in the fuzzy normed space is the most important tool to prove this generalization. Also, as a result and by application, we use the strong law of large numbers for random sets in the fuzzy metric space for the bootstrap mean.
\end{abstract}

\section{Introduction}

The study of the theory of random sets started with Robbins $[1,2]$. Kendall [3], Matheron [4], and Fortet and Kambouzia [5] were among others who studied this field. The motivation for studying random sets is both theoretical and practical. Theoretically, they generalized random variables, random vectors, and fuzzy random variables. Also, practically, they depicted geometrical objects in certain models of growth [6].

The strong law of large numbers (SLLN) for random sets and fuzzy random variables in the Pompeiu-Hausdorff metric and the generalized Pompeiu-Hausdorff metric has been studied since 1982. These studies are based on the embedding theorems, namely, Rådström and Harmender theorems. Note that because the compact subset in the Banach space is not a vector space (with respect to Minkowski addition) in the Pompeiu-Hausdorff metric, proving the SLLN is not easy in this space (see [6]).

The studies in this field began with the Puri and Ralescu [6] in 1983 for random sets in Banach space (Artstein et al. [7] in 1975 and Cressie [8] in 1978 also conducted studies on the SLLN in the Euclidean $p$-dimensional space). Puri and Ralescu [9] in 1986 provided a definition of a fuzzy random variable. In the same year, Kelement et al. [10] established the SLLN for the fuzzy random variable. Random sets and fuzzy random variables in the Pompeiu-Hausdorff metric and generalized Pompeiu-Hausdorff metric $\left(d_{\infty}\right)$ are not separable (see [9]). On the other hand, one of the essential conditions in the SLLN is separability. Also, the previous studies show that the convergence in the metric $d_{\infty}$ is stronger than the convergence in the metric $d_{1}$. In 2002, Proske et al. [11] studied the SLLN in the $d_{\infty}$ metric.

López et al. [12] introduced simple convex random sets. At this time, a new approach was begun to express and prove the SLLN. In this approach, the embedding theorems were not used. Also, since the metric space for compact sets in $d_{\infty}$ is not separable, to solve this problem, simple random sets were used. Colubi et al. [13] derived the SLLN for independent identically distributed (i.i.d) fuzzy random sets by the approximation method that was a result of López's studies ([12]). Also, Molchanov [14] in the same year demonstrated the SLLN for the upper semicontinuous functions with a simpler approach. Li and Ogura [15] presented the SLLN for independent (not necessarily identically distributed) fuzzy set-valued random variables whose base space is a separable Banach space or a Euclidean space, in the sense of the extended Pompeiu-Hausdorff metric.

$\mathrm{Fu}$ and Zhang [16] obtained some SLLN for arrays of row-wise independent (not necessarily identically 
distributed) random compact sets and fuzzy random sets whose underlying spaces are separable Banach spaces. Kim et al. established [17] two types of the SLLN for fuzzy random variables taking values on the space of normal and upper semicontinuous fuzzy sets with compact support in a separable Banach space.

Probabilistic metric spaces were introduced by Menger [18] who generalized the theory of metric spaces. In Menger's theory, the concept of distance is considered to be statistical or probabilistic; i.e., he proposed associating a distribution function with every pair of elements $x, y$ instead of associating a number. Many research studies have been done on probabilistic metric spaces in recent years. The motivation of introducing the probabilistic metric space is the fact that in many situations the distance between two points is inexact rather than a single real number. But when the uncertainty is due to fuzziness rather than randomness, as sometimes in the measurement of an ordinary length, it seems that the concept of a fuzzy metric space is more suitable. The concept of fuzzy metric space introduced by Kramosil and Michalek [19] and George and Veeramani [20] modified this concept.

Puri and Ralescu [9] provided a definition of a fuzzy random variable based on random sets. Now, if we want to use the concept of fuzzy metric space and the SLLN for fuzzy random variables, it is necessary to consider this concept and theorem for random sets. To reach this purpose, we prove an embedding theorem for random sets in fuzzy metric space. Also, we will introduce the generalization of Lebesgue dominated convergence theorem, in the case of random sets in fuzzy metric space.

Efron's bootstrapping [21] is a resampling scheme that is used on a variety of estimation problems. Considering the importance of the SLLN in the bootstrap method, much has been done in this area by various researchers (see Athreya [22], Athreya et al. [23]). In this paper, we generalize the SLLN in the fuzzy metric space for the bootstrap mean.

In Section 2, some preliminaries and lemmas will be presented. In Section 3, the generalized Rådström embedding theorem is expressed. In the next section, the Lebesgue dominated convergence theorem will be generalized. The SLLN in fuzzy metric space is stated in Section 5. In Section 6 , we use the SLLN in fuzzy metric space for the bootstrap mean. The final section is the conclusion.

\section{Preliminaries}

In this section, at first, we define the $t$-norm, the new generalized Hukuhara difference (T-difference), fuzzy metric space, and fuzzy normed space and give several lemmas and theorems in this space that will be used in the next section.

Triangular norms ( $t$-norms for short), introduced by Schweizer and Sklar [24], play a key role in the theory of fuzzy metric spaces. Also, a fuzzy subset of $X$ (fuzzy set) is a function of $u: X \longrightarrow[0,1]$. In the following, the definitions of $t$-norm, fuzzy metric space, and fuzzy normed space and its properties are presented.
Definition 1 (see [25]). A $t$-norm is a binary operation $*:[0,1] \times[0,1] \longrightarrow[0,1]$, such that for all $a, b, c, d \in[0,1]$ the following four axioms are satisfied:

(1) $a * 1=a$

(2) $a * b \leq c * d$ whenever $a \leq c$ and $b \leq d$

(3) $a * b=b * a$

(4) $a *(b * c)=(a * b) * c$

An $i$-norm $*$ is said to be continuous if it is a continuous function on $[0,1] \times[0,1]$.

Let $(\mathscr{X},\|\|$.$) be a separable normed space. Denote by$ $\mathscr{K}(\mathscr{X})$ and $\mathscr{K}_{c}(\mathscr{X})$ the collection of nonempty compact and compact convex subsets of $\mathscr{X}$. The Minkowski addition and scalar multiplication are defined in $\mathscr{K}_{c}(\mathscr{X})$ :

$$
\begin{aligned}
A+B & =\{a+b \mid a \in A, b \in B\}, \\
\lambda A & =\{\lambda a \mid a \in A\},
\end{aligned}
$$

for $A, B \in \mathscr{K}_{c}(\mathscr{X})$ and $\lambda \in \mathbb{R}$. Note that $\mathscr{K}_{c}(\mathscr{X})$ is not a vector space but it becomes a complete metric space when endowed with the Pompeiu-Hausdorff distance. We know that the Pompeiu-Hausdorff distance is defined as

$$
d_{H}(A, B)=\max \left\{\sup _{a \in A} \inf _{b \in B}\|a-b\|, \sup _{b \in B} \inf _{a \in A}\|a-b\|\right\} \text {, }
$$

where $\|$.$\| denotes the norm in \mathscr{X}$ and $A, B \in \mathscr{K}_{c}(\mathscr{X})$ [6]. We use the notation

$$
A=d_{H}(A,\{0\})=\sup \{a \mid a \in A\},
$$

where $A \in \mathscr{K}_{c}(\mathscr{X})$. From here on throughout the article, $d_{H}$ is the Pompeiu-Hausdorff metric.

In (1), if $\lambda=-1$, scalar multiplication gives the opposite $-A=(-1) A=\{-a \mid a \in A\}$ but, in general, $A+(-A) \neq\{0\}$; i.e., the opposite of $A$ is not the inverse of $A$ in Minkowski addition (unless $A=\{a\}$ is a singleton). Minkowski's difference is $A-B=A+(-1) B=\{a-b \mid a \in A, b \in B\}$. This fact is that, in general, even if it is true that $(A+C=B+C) \Longleftrightarrow A=B$, addition/subtraction simplification is not valid, i.e., $(A+B)-B \neq A$. To partially overcome this situation, Hukuhara in [26] introduced the following $\mathrm{H}$-difference:

$$
A \ominus_{H} B=C \Longleftrightarrow A=B+C,
$$

and an important property of $\ominus_{H}$ is that $A \ominus_{H} A=\{0\}$. From an algebraic point of view, the difference of two sets $A$ and $B$ may be interpreted both in terms of addition as in (4) or in terms of negative addition; i.e.,

$$
A \boxminus B=C \Longleftrightarrow B=A+(-1) C,
$$

where $(-1) C$ is the opposite set of $C$. Conditions (4) and (5) are compatible with each other; that is why Stefanini [27] suggested a generalization of the Hukuhara difference as follows.

Definition 2 (see [27]). Let $A, B \in \mathscr{K}_{c}(\mathscr{X})$; we define the generalized Hukuhara difference (gH-difference) of $A$ and $B$ as the set $C \in \mathscr{K}_{c}(\mathscr{X})$ such that 


$$
A \ominus_{g} B=C \Leftrightarrow\left\{\begin{array}{l}
(i) A=B+C, \\
(i i) B=A+(-1) C .
\end{array}\right.
$$

Sometimes, the gH-difference in (6) of $A, B \in \mathscr{K}_{c}\left(\mathbb{R}^{n}\right)$ does not exist (see [27]).

Stefanini and Bede [28] defined a generalized difference for compact convex sets, even if the gH-difference $A \ominus_{g} B$ does not exist. This difference is called the total gH-difference of $A, B$ (T-difference for short). In the following, we introduce some preliminary concepts for compact convex sets which are required to express the T-difference.

Let $\mathbb{S}^{n-1}=\left\{\mathscr{P} \mid \mathscr{P} \in \mathbb{R}^{n},\|\mathscr{P}\|=1\right\}$ be a unit sphere. The support function of $A \in \mathscr{K}_{c}\left(\mathbb{R}^{n}\right)$ is $s_{A}: \mathbb{S}^{n-1} \longrightarrow \mathbb{R}$ defined by

$$
s_{A}(x)=\sup \{\langle x, a\rangle \mid a \in A\}, \quad \forall x \in \mathbb{S}^{n-1},
$$

where $\left(\mathbb{R}^{n},\langle.,\rangle.\right)$ is a (real) Hilbert space with internal product $\langle.,$.$\rangle and associated norm \langle x, x\rangle^{1 / 2}$.

The gH-difference of $A, B \in \mathscr{K}_{c}\left(\mathbb{R}^{n}\right)$ can be expressed by the use of the support functions [28]. Consider $A, B \in \mathscr{K}_{c}\left(\mathbb{R}^{n}\right)$ with $C=A \ominus_{g} B$ as defined in (6); let $s_{A}, s_{B}, s_{C}$, and $s_{-C}$ be the support functions of $A, B, C$, and $(-1) C$, respectively. In case $(i)$, we have $s_{A}=s_{B}+s_{C}$, and in case (ii), we have $s_{B}=s_{A}+s_{-C}$. So, for all $\mathscr{P} \in \mathbb{S}^{n-1}$ [28],

$$
s_{C}(\mathscr{P})=\left\{\begin{array}{l}
s_{A}(\mathscr{P})-s_{B}(\mathscr{P}), \operatorname{incase}(i), \\
s_{-B}(\mathscr{P})-s_{-A}(\mathscr{P}), \operatorname{incase}(i i) .
\end{array}\right.
$$

If $A \in \mathscr{K}_{c}\left(\mathbb{R}^{n}\right)$, then $A$ can be associated with a family of compact intervals that characterize it. For $x \in \mathbb{R}^{n}$, the support function $s_{A}: \mathbb{R}^{n} \longrightarrow \mathbb{R}$ is defined by

$$
s_{A}(x)=\max \{\langle a, x\rangle \mid a \in A\} .
$$

As a dual for the support function, $l_{A}: \mathbb{R}^{n} \longrightarrow \mathbb{R}$ is defined by

$$
l_{A}(x)=\min \{\langle a, x\rangle \mid a \in A\} .
$$

Also, Stefanini and Bede [28] defined for each $\mathscr{P} \in \mathbb{R}^{n}$ the compact intervals

$$
I_{A}(\mathscr{P})=\left[l_{A}(\mathscr{P}), s_{A}(\mathscr{P})\right] .
$$

The following gH-differences for intervals are well defined for all $\mathscr{P} \in \mathbb{S}^{n-1}$ :

$$
\begin{aligned}
I_{A, B}(\mathscr{P}) & =I_{A}(\mathscr{P}) \ominus_{g} I_{B}(\mathscr{P}), \\
I_{B, A}(\mathscr{P}) & =I_{B}(\mathscr{P}) \ominus_{g} I_{A}(\mathscr{P})=-I_{A, B}(\mathscr{P}), \\
I_{A, B}(\mathscr{P}) & =\left[I_{A, B}^{-}(\mathscr{P}), I_{A, B}^{+}(\mathscr{P})\right], \quad \forall \mathscr{P} \in \mathbb{S}^{n-1},
\end{aligned}
$$

where

$$
\begin{aligned}
& I_{A, B}^{-}(\mathscr{P})=\min \left\{l_{A}(\mathscr{P})-l_{B}(\mathscr{P}), s_{A}(\mathscr{P})-s_{B}(\mathscr{P})\right\}, \\
& I_{A, B}^{+}(\mathscr{P})=\max \left\{l_{A}(\mathscr{P})-l_{B}(\mathscr{P}), s_{A}(\mathscr{P})-s_{B}(\mathscr{P})\right\} .
\end{aligned}
$$

Definition 3 (see [28]). Let $A, B \in \mathscr{K}_{c}\left(\mathbb{R}^{n}\right)$ and consider the following family of sets:

$$
\begin{aligned}
\mathbb{D}(A, B)= & \left\{C \mid C \in \mathscr{K}_{c}\left(\mathbb{R}^{n}\right), A \subseteq B+C, B \subseteq A-C\right\} \\
& =\left\{C \mid C \in \mathscr{K}_{c}\left(\mathbb{R}^{n}\right), I_{A, B} \subseteq I_{C}\right\},
\end{aligned}
$$

where $I_{A, B}$ and $I_{C}$ are the interval-valued functions defined in (11) and (12), respectively. The set $\mathbb{D}(A, B)$ will be called the (generic) difference set of the pair $(A, B)$. It is immediate that $\mathbb{D}(B, A)=-\mathbb{D}(A, B)$; i.e., $C \in \mathbb{D}(A, B)$ if and only if $-C \in \mathbb{D}(B, A)$.

The new generalized difference will be defined as an element of the family $\mathbb{D}(A, B)$, by requiring appropriate additional conditions.

Definition 4 (see [28]). $C \in \mathbb{D}(A, B)$ is called minimal with respect to set magnitude (norm-minimal for short) if no $C^{\prime} \in \mathbb{D}(A, B)$ exists with $\left\|C^{\prime}\right\|<\|C\|$.

The set of all elements of $\mathbb{D}(A, B)$ with the normminimality property will be denoted by $\mathbb{D}_{\text {norm }}(A, B)$. It is immediate that $\mathbb{D}_{\text {norm }}(B, A)=-\mathbb{D}_{\text {norm }}(A, B)$. Furthermore, there exists a real number $\alpha(A, B) \geq 0$, depending only on $A$ and $B$, such that

$$
\|C\|=\alpha(A, B) \text { for all } C \in \mathbb{D}_{\text {norm }}(A, B) .
$$

Clearly,

$$
0 \leq \alpha(A, B) \leq\|A-B\|
$$

because $A-B=\mathbb{D}(A, B) a$.

Definition 5 (see [28]). Let $A, B \in \mathscr{K}_{c}\left(\mathbb{R}^{n}\right)$ be given. The following convex set always exists and is unique

$$
A \ominus_{T} B=\operatorname{cl}\left(\operatorname{conv} \cup\left\{C \mid C \in \mathbb{D}_{\text {norm }}(A, B)\right\}\right),
$$

where $\mathrm{cl}$ is the closure of $\mathbb{D}_{\text {norm }}(A, B)$ with respect to convex unions of its elements. $A \ominus_{T} B \in \mathscr{K}_{c}\left(\mathbb{R}^{n}\right)$ has the following basic properties:

(1) $A \subseteq B+A \ominus_{T} B$.

(2) $B \subseteq A-A \ominus_{T} B$.

(3) $A \ominus_{T} B \subseteq A-B$.

(4) $A \ominus_{T} B$ is norm-minimal with respect to $\mathbb{D}(A, B)$.

(5) $A \ominus_{T} B=\{0\}$ if and only if $A=B$.

(6) $B \ominus_{T} A=-\left(A \ominus_{T} B\right)$.

(7) If the gH-difference exists, then $A \ominus_{g} B=A \ominus_{T} B$.

(8) The magnitude of $A \ominus_{T} B$ coincides with the Pompeiu-Hausdorff distance; i.e.,

$$
\left\|A \ominus_{T} B\right\|=\alpha(A, B)=d_{H}(A, B) .
$$

The set $A \ominus_{T} B \in \mathscr{K}_{c}\left(\mathbb{R}^{n}\right)$ will be called the total $\mathrm{gH}$ difference of $A$ and $B$ (T-difference for short).

Definition 6 (see [20]). Let X be an arbitrary nonempty set and $*$ is a continuous t-norm. The 3 -tuple $(\mathrm{X}, M, *)$ is said to be a fuzzy metric space if $M$ is a fuzzy set on $\mathrm{X} \times \mathrm{X} \times$ $(0, \infty)$ satisfying the following conditions for all $x, y, z \in \mathrm{X}$ and $t, s>0$ :

(1) $M(x, y, 0)>0, \forall t>0$

(2) $M(x, y, t)=1, \forall t>0$ if and only if $x=y$ 
(3) $M(x, y, t)=M(y, x, t)$

(4) $M(x, y, t) * M(y, z, s) \leq M(x, z, t+s), \forall t, s>0$

(5) $M(x, y,):.(0, \infty) \longrightarrow[0,1]$ is continuous

Example 1 (see [20]). Let $(\mathfrak{X}, d)$ be a metric space. Define $a * b=a b$ or $a * b=\min \{a, b\}$ and $\forall x, y \in \mathfrak{X}$,

$$
M(x, y, t)=\frac{k t^{n}}{k t^{n}+m d(x, y)}, \quad k, m, n \in \mathbb{R}^{+} .
$$

In this case, $(\mathfrak{X}, M, *)$ is a fuzzy metric space. In particular, if $k=n=m=1$, then

$$
M(x, y, t)=\frac{t}{t+d(x, y)},
$$

which is called the standard fuzzy metric induced by metric d.

Definition 7 (see [20]). A sequence $\left\{x_{n}\right\}$ in a fuzzy metric space $(\mathfrak{X}, M, *)$ is a Cauchy sequence if and only if for each $0<\varepsilon<1$ and $t>0$ there exist $n_{0} \in \mathbb{N}$ such that for all $n, m \geq n_{0}$

$$
M\left(x_{n}, x_{m}, t\right)>1-\varepsilon \text {. }
$$

A fuzzy metric space is said to be complete if and only if every Cauchy sequence is convergent.

Definition 8 (see [29]). The 3 -tuple $(\mathscr{X}, N, *)$ is said to be a fuzzy normed space if $\mathscr{X}$ is a vector space, $*$ is a continuous t-norm, and $N$ is a fuzzy set on $\mathscr{X} \times(0, \infty)$ satisfying the following conditions for every $x, y \in \mathscr{X}$ and $t, s>0$ :

(1) $N(x, t)>0, \forall t>0$

(2) $N(x, t)=1$ if and only if $x=0$

(3) $N(\lambda x, t)=N(x, t /|\lambda|), \forall \lambda \neq 0$

(4) $N(x, t) * N(y, s) \leq N(x+y, t+s), \forall t, s>0$

(5) $N(x,):.(0, \infty) \longrightarrow[0,1]$ is continuous

(6) $\lim _{t \rightarrow \infty} N(x, t)=1$

Definition 9. Let $(\mathscr{X},\|\|$.$) be a normed space. Also, addition,$ multiplication, distance, norm, and difference are defined in (1)-(3) and (6), respectively. If $A \in \mathscr{K}_{c}(\mathscr{X})$, then $N_{d_{H}}(A, t)=\phi(\|A\|, t)$ is a fuzzy norm, where $\phi(., t)$ is a decreasing function with respect to $\|$.$\| . It is called a fuzzy$ norm induced by $\|$.$\| .$

It is immediate that $\left(\mathscr{X}, N_{d_{H}}, *\right)$ is a fuzzy normed space where $*$ is a continuous $t$-norm.

Example 2 (see [29]). Let $(\mathscr{X},\|\|$.$) be a normed space.$ Suppose that $a * b=a b$ or $a * b=\min \{a, b\}$, and $\forall A \in \mathscr{K}_{c}(\mathscr{X})$,

$$
N_{d_{H}}(A, t)=\frac{k t^{n}}{k t^{n}+m\|A\|}, \quad k, m, n \in \mathbb{R}^{+},
$$

where $\|A\|$ is defined in (3). In this case, $\left(\mathscr{X}, N_{d_{H}}, *\right)$ is a fuzzy normed space.
Lemma 1. Let $\left(\mathscr{X}, N_{d_{H}}\right.$, $)$ be a fuzzy normed space and $A, B \in \mathscr{K}_{c}(\mathscr{X})$. If we define

$$
M_{d_{H}}(A, B, t)=N_{d_{H}}\left(A \ominus_{T} B, t\right),
$$

Then, $M_{d_{H}}$ is a fuzzy metric on $\mathscr{X}$, which is called the fuzzy metric induced by the fuzzy norm $N_{d_{H}}$.

Proof. According to Definitions 3 and 4 and Lemma 3 in [29], it is easy to show that Lemma 1 is established.

Lemma 2. A fuzzy metric $M_{d_{H}}$, which is induced by a fuzzy norm $N_{d_{H}}$, has the following properties for all $A, B, C \in \mathscr{K}_{c}(\mathscr{X})$ and every scalar $\lambda \neq 0$ :

(1) $M_{d_{H}}(A+C, B+C, t)=M_{d_{H}}(A, B, t)$

(2) $M_{d_{H}}(\lambda A, \lambda B, t)=M_{d_{H}}(A, B, t /|\lambda|)$.

Proof. By Lemma 1, Definition 9, and Lemma 4 [29], it is easy to show that the result is established; for example, for (2), we have

$$
\begin{aligned}
M_{d_{H}}(\lambda A, \lambda B, t) & =N_{d_{H}}\left(\lambda A \ominus_{T} \lambda B, t\right)=N_{d_{H}}\left(A \ominus_{T} B, t /|\lambda|\right) \\
& =M_{d_{H}}(A, B, t /|\lambda|) .
\end{aligned}
$$

\section{Generalized Rådström Embedding Theorem}

As mentioned in Section 1, the compact subset in the Banach space is not a vector space (with respect to Minkowski addition) in the Pompeiu-Hausdorff metric (see [6]). The Rådström embedding theorem states that the collection of nonempty closed bounded and convex subsets of a Banach space can be embedded in a normed space. This theorem enables us to prove the SLLN.

In the following, first, we present the properties of the fuzzy metric space $\mathscr{K}_{c}(\mathscr{X})$ in the fuzzy metric $M_{d_{H}}$ and then generalize the Rådström embedding theorem.

Theorem 1. Let $\left(\mathscr{X}, N_{d_{H}}\right.$, $)$ be a fuzzy normed space and $M_{d_{H}}$ is fuzzy metric induced by $N_{d_{H}}$. Suppose that $\mathscr{K}_{c}(\mathscr{X})$ is the collection of nonempty compact convex subsets of $\mathscr{X}$; then, $\left(\mathscr{K}_{c}(\mathscr{X}), M_{d_{H}}, *\right)$ is a fuzzy metric space.

Proof. By using Definition 9 and T-difference, we show that for all $A, B, C \in \mathscr{K}_{c}(\mathscr{X})$, the conditions of Definition 6 are established as follows:

(1) $M_{d_{H}}(A, B, t)=N_{d_{H}}\left(A \ominus_{T} B, t\right)>0, \forall t>0$

(2) $A=B$ if and only if $M_{d_{H}}(A, B, t)=N_{d_{H}}\left(A \ominus_{T} B, t\right)=$ $N_{d_{H}}(\{0\}, t)=1$

(3) $M_{d_{H}}(A, B, t)=N_{d_{H}}\left(A \ominus_{T} B, t\right)=N_{d_{H}}\left(B \ominus_{T} A, t\right)=$ $M_{d_{H}}(B, A, t)$

(4) $\forall A, B, C \in \mathscr{K}_{c}(\mathscr{X})$ and $t, s>0$

$$
\begin{aligned}
& \leq N_{d_{H}}\left(A \ominus_{T} B+B \ominus_{T} C, t+s\right) \\
& \leq N_{d_{H}}\left(A \ominus_{T} C, t+s\right)
\end{aligned}
$$




$$
=M_{d_{H}}(A, C, t+s)
$$

(5) $M_{d_{H}}(A, B,)=.N_{d_{H}}\left(A \ominus_{T} B,.\right):(0, \infty) \longrightarrow[0,1]$ is continuous

Rådström in [30] showed that $\mathscr{K}_{c}(\mathscr{X})$, a class of compact convex sets, can be embedded isometrically into normed space. In the following, we will show that this property is established also into a fuzzy normed space.

The space $\mathscr{K}_{c}(\mathscr{X})$ plays an important role since it can be embedded isometrically into a fuzzy normed space. Actually, this theorem generalizes the Rådström embedding theorem [30] from $\mathscr{K}_{c}(\mathscr{X})$ into a fuzzy normed space.

Theorem 2. Let $\mathscr{X}$ be a separable normed space. There exist a fuzzy normed space $\chi$ and a function $j: \mathscr{K}_{c}(\mathscr{X}) \longrightarrow \chi$ with the following properties:
(1) $M_{d_{H}}(A, B, t)=M_{d_{H}}(j(A), j(B), t)$
(2) $j(A+B)=j(A)+j(B)$
(3) $j(\lambda A)=\lambda j(A), \forall \lambda \geq 0$

Note that fuzzy normed space $\chi$ is not complete in general, but one can always take the completion of $\chi$, and thus, $\mathscr{K}_{c}(\mathscr{X})$ is embedded into a fuzzy normed space by $j(\cdot)$.

Proof. Since $\mathscr{K}_{c}(\mathscr{X})$ is a class of compact convex sets in normed space, for all $A, B, C \in \mathscr{K}_{c}(\mathscr{X})$ except 3 and $8-10$, all the conditions of the Rådström embedding theorem in [30] are established. Now, we prove 3 and $8-10$ conditions (with $M_{d_{H}}$ instead of $d_{H}$ ).

In Theorem 1 , we showed that $\mathscr{K}_{c}(\mathscr{X})$ with $M_{d_{H}}$ is metrizable. Furthermore, from Lemma 2 in [30], condition 3 is confirmed.

Also, given that $M_{d_{H}}$ is a decreasing function with respect to $d_{H}$, from Theorem 2 in [30], 8-10 conditions in $M_{d_{H}}$ are established. So, there are $\chi$ and $j: \mathscr{K}_{c}(\mathscr{X}) \longrightarrow \chi$.

Properties 2 and 3 follow from the definitions.

\section{Generalized Lebesgue Convergence Theorem}

An important tool in Section 5, which is used to prove the SLLN for random sets in fuzzy metric space, is the Lebesgue convergence theorem. In this section, after defining a random set, we will generalize this theorem for random sets in the fuzzy metric space.

Suppose that $(\Omega, \mathscr{A}, P)$ is a probability space. The following definitions describe the concept of the random closed set, random compact, and random compact convex set.

Definition 10 (see [31]). Let $\mathfrak{C}(\mathscr{X})$ be the family of closed subsets of $\mathscr{X}$. A map $X: \Omega \longrightarrow \mathfrak{C}(\mathscr{X})$ is called a random closed set if, for every $K \in \mathscr{K}(\mathscr{X}),\{\omega: X \cap K \neq \varnothing\} \in \mathscr{A}$.

Definition 11 (see [31]). A random closed set $X$ with almost everywhere compact values (so that $X \in \mathscr{K}(\mathscr{X})$ a.e.) is called a random compact set.

The $\mathscr{X}$-valued random set (i.e., random sets whose values are compact subsets of $\mathscr{X}$ ) is a Borel measurable function $X: \Omega \longrightarrow \mathscr{K}(\mathscr{X})$.
Definition 12 (see [31]). Let $\mathrm{B}(\mathscr{X})$ be Borel $\sigma$-algebra. Random closed sets $X_{1}, \ldots, X_{n}$ are said to be independent if

$$
P\left(X_{1} \in A_{1}, \ldots, X_{n} \in A_{n}\right)=\prod_{i=1}^{n} P\left(X_{i} \in A_{i}\right)
$$

for all $A_{1}, \ldots, A_{n} \in \mathrm{B}(\mathscr{X})$.

For more information about this concept, see [31].

A random closed set $X$ in the separable normed space $\mathscr{X}$ is called integrably bounded if

$$
\|X\|=\sup \{\|x\| \mid x \in X\}
$$

has a finite expectation [31]. In other words, $E X<\infty$. The expected value of the random set was defined by Aumann [32] and later by Debreu [33]. These definitions were shown to be equivalent to Byrne [34]. If $X$ is a random compact set, then $E X$ is defined as

$$
E X=\int_{\Omega} X d P=\left\{\int_{\Omega} f d P ; f \in L^{1}(\Omega, \mathscr{A}, P), f(\omega) \in X(\omega) \text { a.e }\right\} .
$$

Here, $f: \Omega \longrightarrow \mathscr{X}$ is a selection of $X$ and $E f$ denotes the classical expectation (via the Bochner integral). In general, $E X$ may be empty, but if $E X<\infty$, then $E X \in \mathscr{K}(\mathscr{X})$ (Aumann [32], Debreu [33]).

Note that $X$ is a random compact convex set, whenever $X \in \mathscr{K}_{c}(\mathscr{X})$. In the following, the random compact convex set is called a random set for brevity.

In the following, we will introduce the generalized Lebesgue dominated convergence type theorem in the case of random sets in fuzzy metric space, which is used in the next section.

The almost everywhere convergence of random sets is usually defined with respect to the Pompeiu-Hausdorff metric as $d_{H}\left(X_{n}, X\right) \longrightarrow 0$ [31]. It can equally be said $X_{n} \longrightarrow X$ a.e. In fuzzy metric $M_{d_{H}}$ whenever, $M_{d_{H}}\left(X_{n}, X, t\right) \longrightarrow 1$ a.e.

Theorem 3. Let $\left\{X_{k} \mid k \geq 1\right\}$ and $X$ be random sets with values in $\mathscr{K}_{c}(\mathscr{X})$ such that $E\left\|X_{k}\right\|<\infty$ and $E\|X\|<\infty$. Assume that $X_{k}$ in the fuzzy metric $M_{d_{H}}$ is convergent a.e. to $X$ and $d_{H}\left(X_{k}(\omega),\{0\}\right)<h(\omega)$ for all $k \geq 1$, where $h: \Omega \longrightarrow \mathbb{R}$ is integrable. Then, in the fuzzy metric $M_{d_{H}}$,

$$
E X_{k} \longrightarrow E X \text {. }
$$

Proof. Using the inequality in Debreu [33], p.366-367, we have

$$
d_{H}\left(E X_{k}, E X\right) \leq E d_{H}\left(X_{k}, X\right) .
$$

We know that $d_{H}\left(X_{k}, X\right)$ is a random variable, so, $E d_{H}\left(X_{k}, X\right)$ will be real. Therefore, according to Definition 11 and Lemma 1, we have

$$
M_{d_{H}}\left(E X_{k}, E X, t\right) \geq M_{E d_{H}}\left(X_{k}, X, t\right) .
$$

On the other hand, from the hypothesis, since $M_{d_{H}}\left(X_{k}, X, t\right) \longrightarrow 1$ a.e., so, $d_{H}\left(X_{k}, X\right) \longrightarrow 0$ a.e. By the triangle inequality, we get 


$$
d_{H}\left(X_{k}, X\right) \leq d_{H}\left(X_{k},\{0\}\right)+d_{H}(X,\{0\}) \leq h+d_{H}(X,\{0\}) .
$$

According to the integrability $h$ and $X \in \mathscr{K}_{c}(\mathscr{X})$, $d_{H}\left(X_{k}, X\right)$ is integrable. Now, due to the classical Lebesgue dominated convergence theorem

$$
E d_{H}\left(X_{k}, X\right) \longrightarrow 0
$$

therefore

$$
M_{E d_{H}}\left(X_{k}, X, t\right) \longrightarrow 1 \text {. }
$$

Furthermore, from (20), it follows $d_{H}\left(E X_{k}, E X\right) \longrightarrow 0$ a.e. Also, by using (21), we conclude that

$$
M_{d_{H}}\left(E X_{k}, E X, t\right) \longrightarrow 1 \text {. }
$$

This means that (19) holds.

\section{Strong Law of Large Numbers in Fuzzy Metric Space}

In this section, by using the Rådström embedding theorem, we establish the SLLN for random sets in fuzzy Banach space. In the following, we first express the definition of the fuzzy Banach space and then will show that $\left(\mathscr{K}_{c}(\mathscr{F}), M_{d_{H}}, *\right)$ is a separable fuzzy metric space. In the last step, the SLLN will be expressed and proved in the fuzzy Banach space.

Definition 13 (see [29]). The fuzzy normed space $(\mathscr{F}, N, *)$ is said to be a fuzzy Banach space whenever $\mathscr{F}$ is complete with respect to the fuzzy metric induced by a fuzzy norm.

Theorem 4. Fuzzy metric space $\left(\mathscr{K}(\mathscr{F}), M_{d_{H}}, *\right)$ is separable.

Proof. We know that $\left(\mathscr{K}(\mathscr{F}), d_{H}\right)$ is a separable metric space [35], and given that the continuous image of a separable metric space is separable, so, the result is established.

Corollary 1. Fuzzy metric space $\left(\mathscr{K}(\mathscr{F}), M_{d_{H}}\right.$, $)$ is separable, too.

Theorem 5. Let $\left\{X_{k} \mid k \geq 1\right\}$ be independent and identically distributed (i.i.d.) random sets such that $X_{1}$ is integrable. Then,

$$
\frac{\sum_{k=1}^{n} X_{k}}{n} \longrightarrow E\left(X_{1}\right) \text {, a.e. }
$$

the convergence being in the fuzzy metric $M_{d_{H}}$.

Proof. Let $j: \mathscr{K}_{c}(\mathscr{F}) \longrightarrow \chi$ be the isometry provided by the Rådström embedding theorem. Since $\left(\mathscr{K}_{c}(\mathscr{F}), M_{d_{H}}, *\right)$ is separable (Corollary 1), by considering Lemma 1, it is easy to show that fuzzy normed space $\chi$ is separable. Then, $\left\{j\left(X_{k}\right) \mid k \geq 1\right\}$ are i.i.d. $\chi$-valued random elements, where $j$ is an isometry. By a standard SLLN in Banach space, it follows that

$$
\frac{1}{n} \sum_{k=1}^{n} j\left(X_{1}\right) \longrightarrow E\left(j\left(X_{1}\right)\right) \text {, a.e. }
$$

The main point now is to show that $E\left(j\left(X_{1}\right)\right)=j\left(E\left(X_{1}\right)\right)$, if $X_{1}$ is integrable.

Assume first that $X_{1}$ is a simple function (for some $l$ ); i.e.,

$$
X_{1}=\sum_{i=1}^{l} x_{i} I_{A_{i}} x_{i} \in \mathscr{K}_{c}(\mathscr{F}), \quad A_{i} \in \mathscr{A} .
$$

It is easy to check that $E\left(j\left(X_{1}\right)\right)=j\left(E\left(X_{1}\right)\right)$ in this case. To prove this, due to Theorem 1 , we see that

$$
\begin{aligned}
E\left(j\left(X_{1}\right)\right) & =E\left(j\left(\sum_{i=1}^{l} x_{i} I_{A_{i}}\right)\right) \\
& =E\left(\sum_{i=1}^{l} j\left(x_{i}\right) I_{A_{i}}\right) \\
& =\sum_{i=1}^{l} j\left(x_{i}\right) P\left(A_{i}\right) \\
& =j\left(\sum_{i=1}^{l}\left(x_{i}\right) P\left(A_{i}\right)\right) \\
& =j E\left(\sum_{i=1}^{l}\left(x_{i}\right) I_{A_{i}}\right) \\
& =j\left(E\left(X_{1}\right)\right) .
\end{aligned}
$$

Since $X_{1}$ is measurable, there exists a sequence of simple functions $S_{m}$ with $S_{m} \longrightarrow X_{1}$ a.e. in the fuzzy metric $M_{d_{H}}$. Also from the continuity of $M_{d_{H}}$, a.e.

$$
M_{d_{H}}\left(S_{m},\{0\}, t\right) \longrightarrow M_{d_{H}}\left(X_{1},\{0\}, t\right) .
$$

We now consider the truncated random sets $t_{m}$ as follows $(m \longrightarrow \infty)$ :

$t_{m}(\omega)= \begin{cases}S_{m}(\omega), & M_{d_{H}}\left(S_{m}(\omega)\right),\{0\}, t \geq M_{d_{H}}\left(X_{1}(\omega),\{0\}, \frac{t}{2}\right), \\ \{0\}, & \text { o.w. }\end{cases}$

Note that $t_{m}$ are simple functions. It is easy to see in fuzzy metric $M_{d_{H}}$ that $t_{m} \longrightarrow X_{1}$ a.e. and that

$$
M_{d_{H}}\left(t_{m}(\omega),\{0\}, t\right) \geq M_{d_{H}}\left(X_{1}(\omega),\{0\}, \frac{t}{2}\right) .
$$

The hypotheses of Theorem 4 are satisfied, because $t_{m} \longrightarrow X_{1}$, so $E\left(t_{m}\right) \longrightarrow E\left(X_{1}\right)$. Therefore, in $\chi$,

$$
j\left(E\left(t_{m}\right)\right) \longrightarrow j\left(E\left(X_{1}\right)\right) \text {. }
$$

It is easy to see that $j\left(t_{m}\right) \longrightarrow j\left(X_{1}\right)$ and, from properties of the Bochner integral, that $E\left(j\left(t_{m}\right)\right) \longrightarrow E\left(j\left(X_{1}\right)\right)$. Since $j\left(E\left(t_{m}\right)\right)=E\left(j\left(t_{m}\right)\right)$, it follows that $j\left(E\left(X_{1}\right)\right)=E\left(j\left(X_{1}\right)\right)$. Therefore, 


$$
M_{d_{H}}\left(\frac{1}{n} \sum_{k=1}^{n} j\left(X_{k}\right) \ominus_{g} j\left(E\left(X_{1}\right)\right),\{0\}, t\right) \longrightarrow 1 \text {, a.e. }
$$

From the properties of $j$, it follows that

$$
M_{d_{H}}\left(\frac{1}{n} \sum_{k=1}^{n} X_{k} E\left(X_{1}\right) t\right) \longrightarrow 0 \text {, a.e. }
$$

\section{Bootstrap Mean in Fuzzy Metric Space}

The bootstrap method introduced in Efron [21] is a very general resampling procedure for estimating the distributions of statistics based on independent observations. Athreya [22] provided strong Law for the Bootstrap and Athreya et al. [23] established laws of large numbers for bootstrapped U-statistics. Also, Csrgo [36] presented the weak and strong law of large numbers for bootstrap sample means under minimal moment conditions. In this section, we want to generalize the SLLN in fuzzy metric space for the bootstrap mean.

Let $\left\{X_{k} \mid k \geq 1\right\}$ be an infinite sequence of i.i.d. random sets defined on a probability space $(\Omega, \mathscr{A}, P)$ and $X_{1}$ is integrable. For each $n=1,2, \ldots$, let $Y_{n, 1}, Y_{n, 2}, \ldots, Y_{n, m(n)}$ be the ordinary Efron bootstrap sample of size $m(n)$ where $\{m(n)\}$ is a sequence of positive integers. The variables $Y_{n, 1}, Y_{n, 2}, \ldots, Y_{n, m(n)}$ result from sampling $m$ times the sequence $\left\{X_{1}, X_{2}, \ldots, X_{n}\right\}$ with replacement such that at each stage any one element has a probability $1 / n$ to be picked [36].

Suppose that $\bar{X}_{n}^{*}$ is the bootstrap sample mean where

$$
\bar{X}_{n}^{*}=\frac{1}{m(n)} \sum_{i=1}^{m(n)} Y_{n, i} .
$$

Puri and Ralescu [35] provided limit theorems for random compact sets in Banach space. In fact, they showed that

$$
d_{H}\left(\frac{1}{n} \sum_{i=1}^{n} X_{i} E\left(X_{1}\right)\right) \longrightarrow 0 \text {, a.e. }
$$

Now, if we apply this theorem for bootstrap mean, then we have

$$
d_{H}\left(\frac{1}{m(n)} \sum_{i=1}^{m(n)} Y_{n, i} E\left(X_{1}\right)\right) \longrightarrow 0 \text {, a.e. }
$$

Sometimes, the expert's opinion may be important in determining the magnitude or smallness of the distance. In this case, the use of the SLLN in fuzzy metric space (stated in Section 5) is more appropriate. By specifying the value of $t$, the expert can apply his opinion on the distance between two values. In the following, we will illustrate with an example that the bootstrap SLLN in fuzzy metric space is established. In other words,

$$
M_{d_{H}}\left(\frac{1}{m(n)} \sum_{i=1}^{m(n)} Y_{n, i} E\left(X_{1}\right), t\right) \longrightarrow 1, \text { a.e. }
$$

Example 3. The probability that someone in time $A_{1}$ will sell a piece of property at a profit of range $[-1,-0.6]$ thousand dollars is 0.2 , the probability that he in time $\mathrm{A}_{2}$ will sell it at a profit of range $[-0.5,0.5]$ thousand dollars is 0.25 , the probability that in time $\mathrm{A}_{3}$ the sale profit is in the range of $[1.25,1.75]$ is equal to 0.25 , the probability that in time $\mathrm{A}_{4}$ the sale profit is in the range of $[1.8,2.4]$ is equal to 0.15 , and the probability that he in time $\mathrm{A}_{5}$ will profit of range $[2.5,3.5]$ is 0.15 . What is his expected profit?

Here, we denote the random set with $G(\omega)$ :

$$
G(\omega)= \begin{cases}{[-1,-0.6],} & \omega \in A_{1}, \\ {[-0.5,0.5],} & \omega \in A_{2}, \\ {[1.25,1.75],} & \omega \in A_{3}, \\ {[1.8,2.4],} & \omega \in A_{4}, \\ {[2.5,3.5],} & \omega \in A_{5} .\end{cases}
$$

Then, for each random set, the Aumann integral (19) is denoted by $E[G]$ and is equal to

$$
E(G)=\int_{\Omega} G d P=\left\{\int_{A_{1}} f d P+\ldots+\int_{A_{5}} f d P ; f \in L^{1}(\Omega, \mathscr{A}, P), f(\omega) \in G(\omega), \text { a.e }\right\}=[0.6325,1.3275]
$$

Suppose that the following data are a random sample from the population:

$$
\left\{\begin{array}{c}
{[1.25,1.75],[-1,-0.6],[-0.5,0.5],[1.8,2.4]} \\
{[-0.5,0.5],[2.5,3.5],[-0.5,0.5],[1.25,1.75],[2.5,3.5],[-1,-0.6]}
\end{array}\right\}
$$

Now, we generate 1000 samples by using the bootstrap method. In the next step, we calculate the mean of every 1000 samples. To show better convergence, we compute the mean of the bootstrap method for the number of different iterations with ten steps in the interval from 1 to 1000 . In other words, we calculate the mean value in steps $10,20, \ldots$, 1000 and display it on the chart. These values are shown in Figure 1. 


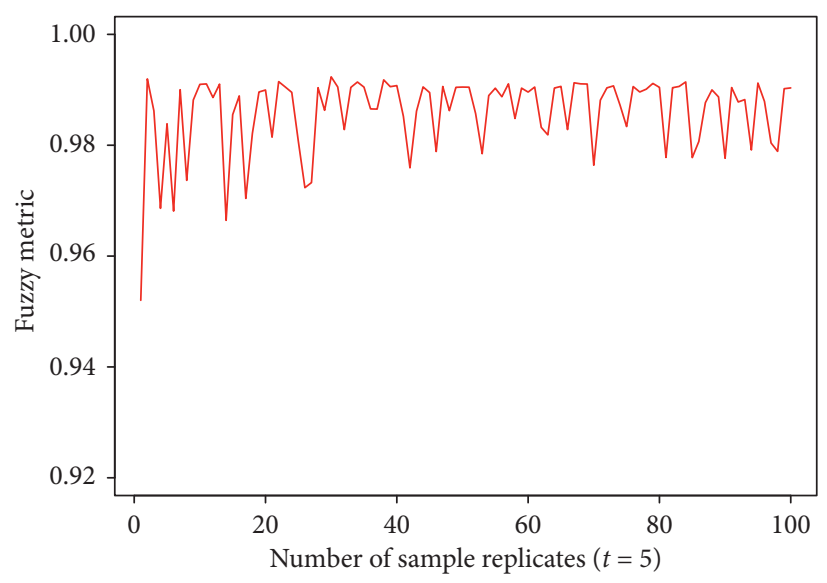

Figure 1: $M_{d_{H}}$ when $m(n) \longrightarrow \infty$ and $t=5$.

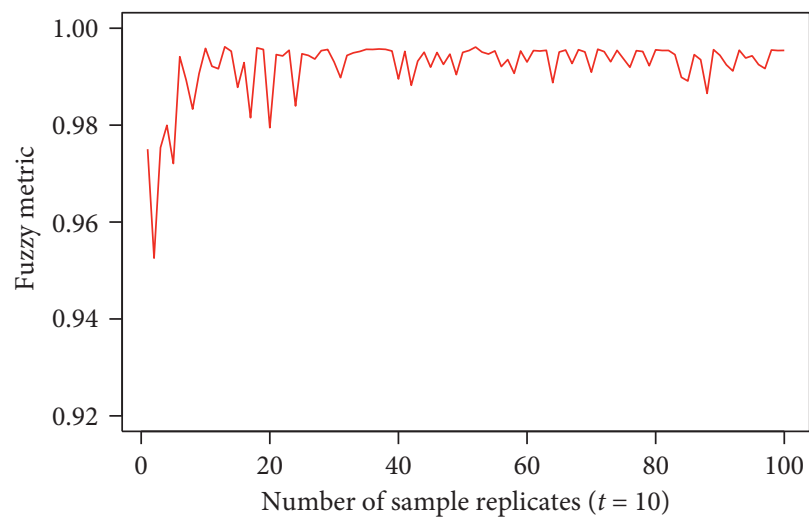

(a)

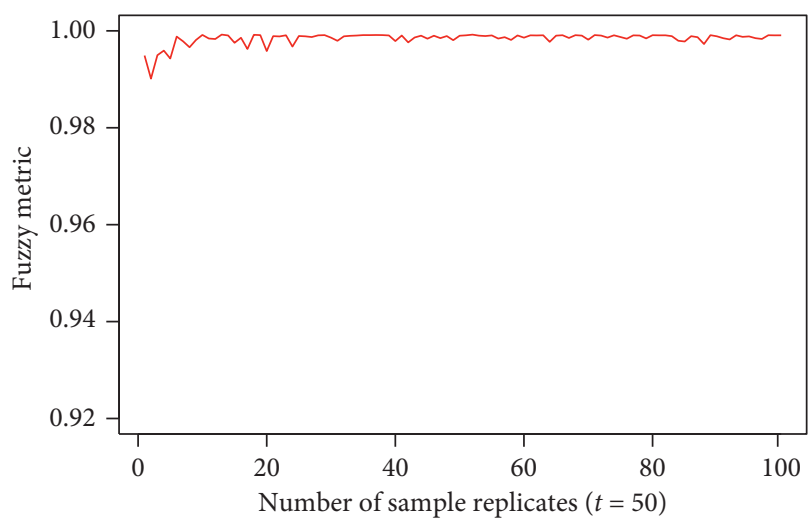

(c)

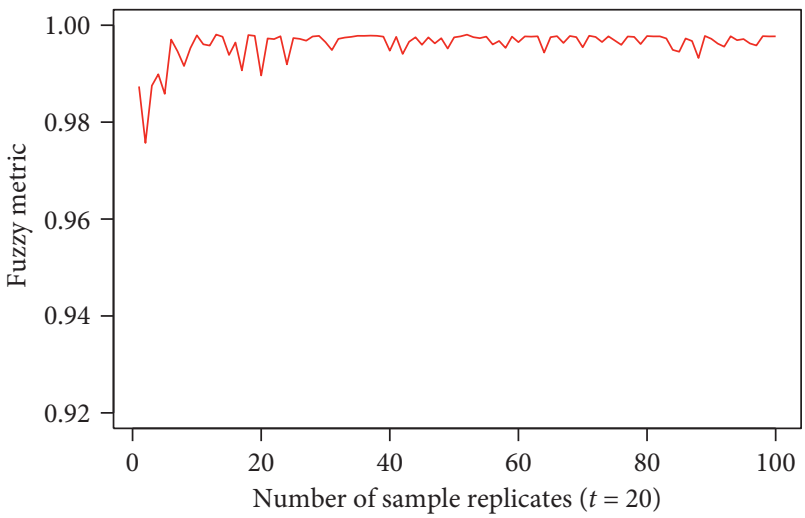

(b)

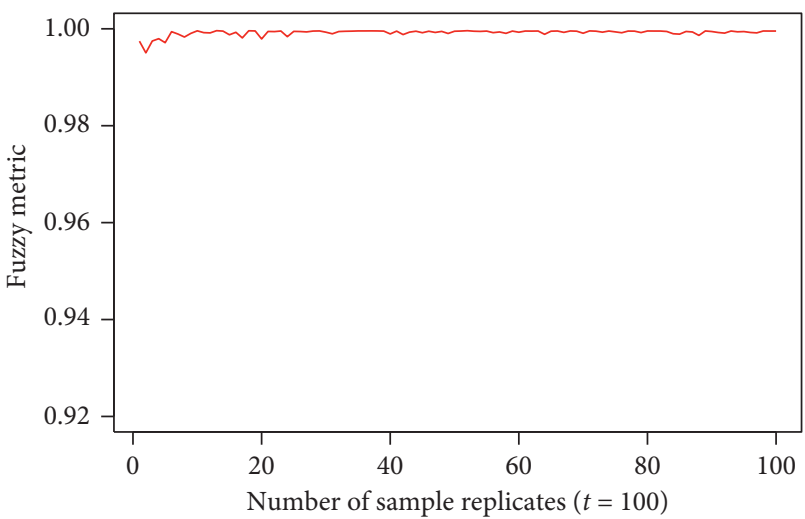

(d)

Figure 2: Behavior of $M_{d_{H}}$ when $m(n) \longrightarrow \infty$ for different values of $t(t=10, t=20, t=50, t=100)$.

As we can see in Figure 1, when $m(n) \longrightarrow \infty$ and $t=5$, the expectation of the random set tends to the sample mean by using the bootstrap method in the fuzzy metric. In other words,

$$
M_{d_{H}}\left(\frac{1}{m(n)} \sum_{i=1}^{m(n)} Y_{n, i} E\left(X_{1}\right), t\right) \longrightarrow 1 \text {, a.e. }
$$

Also, the effect of value $t$ on the behavior of $M_{d_{H}}$ and convergence rate can be seen in Figure 2. In fact, using a low value of $t$ reduces the value of $M_{d_{H}}$ to 0 too quickly as the Pompeiu-Hausdorff distance increases. On the other hand, if a high value is used, the value of $M_{d_{H}}$ decreases too slowly (Figure 2). The expert's opinion is important in determining the appropriate value of $t$. 


\section{Conclusion}

When the uncertainty is fuzziness, as sometimes in the measurement of an ordinary length, the concept of a fuzzy metric space is more suitable. Since the fuzzy random variable is defined on the basis of a random set, the SLLN for random sets in fuzzy metric space assists us in expressing this theorem for fuzzy random variables in a fuzzy metric space. Therefore, we have presented a new theorem for the study of the SLLN for random sets in fuzzy metric spaces in the sense of George and Veeramani [20]. Also, we generalized the Rådström embedding theorem and Lebesgue dominated convergence that are important tools to prove these theorems. In fact, this article can provide the conditions for the expression of limit theorems for fuzzy random variables in fuzzy metric space. As an application of the SLLN for random sets in fuzzy metric space, we show that $1 / m(n) \sum_{i=1}^{m(n)} Y_{n, i}$ tends to $E\left(X_{1}\right)$ in fuzzy metric space for bootstrap method. In fact, when an expert's opinion is important in determining distance, the value of $t$ in fuzzy metric becomes significant.

\section{Data Availability}

This research is a theoretical study, and no data were used to support this study.

\section{Conflicts of Interest}

The authors declare that they have no conflicts of interest.

\section{References}

[1] H. E. Robbins, "On the measure of a random set," The Annals of Mathematical Statistics, vol. 15, no. 1, pp. 70-74, 1944.

[2] H. E. Robbins, "On the measure of a random set. ii," The Annals of Mathematical Statistics, vol. 16, no. 4, pp. 342-347, 1945.

[3] D. G. Kendall, "Foundation of a theory of random sets," Stochastic Geometry, vol. 10, pp. 322-376, 1974.

[4] G. Matheron, Random Sets and Integral Geometry, Wiley, New York, NY, USA, 1975.

[5] R. Fortet and M. Kambouzia, "Ensembles aléatoires induits par une répartition ponctuelle aléatoire," The Annals of Statistics, vol. 280, pp. 1447-1450, 1975.

[6] M. L. Puri and D. A. Ralescu, "Strong law of large numbers for banach space valued random sets," The Annals of Probability, vol. 11, no. 1, pp. 222-224, 1983.

[7] Z. Artstein and J. C. Hansen, "Convexification in limit laws of random sets in banach spaces," The Annals of Probability, vol. 13, no. 1, pp. 307-309, 1985.

[8] N. Cressie, "A strong limit theorem for random sets," Advances in Applied Probability, vol. 10, pp. 36-46, 1978.

[9] M. L. Puri and D. A. Ralescu, "Fuzzy random variables," Journal of Mathematical Analysis and Applications, vol. 114, no. 2, pp. 409-422, 1986.

[10] E. Klement, M. L. Puri, and D. Ralescu, "Limit theorems for fuzzy random variables," The Royal Society, vol. 21, pp. 171$182,1986$.

[11] F. N. Proske and M. L. Puri, "Strong law of large numbers for banach space valued fuzzy random variables," Journal of Theoretical Probability, vol. 15, no. 2, pp. 543-551, 2002.
[12] M. López-Díaz and M. A. Gil, “Approximating integrably bounded fuzzy random variables in terms of the "generalized" Hausdorff metric," Information Sciences, vol. 104, no. 3-4, pp. 279-291, 1998.

[13] A. Colubi, M. López-Díiaz, J. S. Domíinguez-Menchero, and M. A. Gil, "A generalized strong law of large numbers," Probability Theory and Related Fields, vol. 114, no. 3, pp. 401-417, 1999.

[14] I. S. Molchanov, "On strong laws of large numbers for random upper semicontinuous functions," Journal of Mathematical Analysis and Applications, vol. 235, no. 1, pp. 349-355, 1999.

[15] S. Li and Y. Ogura, "Strong laws of large numbers for independent fuzzy set-valued random variables," Fuzzy Sets and Systems, vol. 157, no. 19, pp. 2569-2578, 2006.

[16] K.-A. Fu and L.-X. Zhang, "Strong laws of large numbers for arrays of rowwise independent random compact sets and fuzzy random sets," Fuzzy Sets and Systems, vol. 159, no. 24, pp. 3360-3368, 2008.

[17] J.-M. Kim and Y. K. Kim, "Some notes on strong law of large numbers for banach space valued fuzzy random variables," Korean Journal of Mathematics, vol. 21, no. 4, pp. 383-399, 2013.

[18] K. Menger, "Statistical metrics," Proceedings of the National Academy of Sciences, vol. 28, no. 12, pp. 535-537, 1942.

[19] I. Kramosil and J. Michálek, "Fuzzy metrics and statistical metric spaces," Kybernetika, vol. 11, no. 5, pp. 336-344, 1975.

[20] A. George and P. Veeramani, "On some results in fuzzy metric spaces,” Fuzzy Sets and Systems, vol. 64, no. 3, pp. 395-399, 1994.

[21] B. Efron, "Bootstrap methods: another look at the jackknife," The Annals of Statistics, vol. 7, no. 1, pp. 1-26, 1979.

[22] K. B. Athreya, "Strong law for the bootstrap," Statistics \& Probability Letters, vol. 1, no. 3, pp. 147-150, 1983.

[23] K. B. Athreya, M. Ghosh, L. Y. Low, and P. K. Sen, "Laws of large numbers for bootstrappedU-statistics," Journal of Statistical Planning and Inference, vol. 9, no. 2, pp. 185-194, 1984.

[24] B. Schweizer and A. Sklar, "Statistical metric spaces," Pacific Journal of Mathematics, vol. 10, no. 1, pp. 313-334, 1960.

[25] E. P. Klement, R. Mesiar, and E. Pap, Triangular Norms, Springer Science, Berlin, Germany, 2013.

[26] M. Hukuhara, "Integration des applications mesurables dont la valeur est uncompact convexe," Funkcialaj Ekvacioj, vol. 10, no. 3, pp. 205-223, 1967.

[27] L. Stefanini, "A generalization of hukuhara difference and division for interval and fuzzy arithmetic," Fuzzy Sets and Systems, vol. 161, no. 11, pp. 1564-1584, 2010.

[28] L. Stefanini and B. Bede, "A new gH-difference for multidimensional convex sets and convex fuzzy sets," Axioms, vol. 8, no. 2, p. 48, 2019.

[29] R. Saadati and S. Vaezpour, "Some results on fuzzy banach spaces," Journal of Applied Mathematics and Computing, vol. 17, no. 1-2, pp. 475-484, 2005.

[30] H. Rådström, "An embedding theorem for spaces of convex sets," Proceedings of the American Mathematical Society, vol. 3, no. 1, pp. 165-169, 1952.

[31] I. Molchanov and I. S. Molchanov, Theory of Random Sets, Springer, Berlin, Germany, 2005.

[32] R. J. Aumann, "Integrals of set-valued functions," Journal of Mathematical Analysis and Applications, vol. 12, no. 1, pp. 1-12, 1965.

[33] G. Debreu, "Integration of correspondences," Proceedings of the Fifth Berkeley Symposium on Mathematical Statistics and Probability: Contributions to Probability Theory, Part 12, pp. 351-372, 1967. 
[34] C. L. Byrne, "Remarks on the set-valued integrals of debreu and aumann," Journal of Mathematical Analysis and Applications, vol. 62, no. 2, pp. 243-246, 1978.

[35] M. L. Puri and D. A. Ralescu, "Limit theorems for random compact sets in Banach space," Mathematical Proceedings of the Cambridge Philosophical Society, vol. 97, no. 1, pp. 151$158,1985$.

[36] S. Csrgo, "On the law of large numbers for the bootstrap mean," Statistics Probability Letters, vol. 14, no. 1, pp. 1-7, 1992. 